\title{
Comparing Two Binned Probability Distributions for Information Access Evaluation
}

\author{
Tetsuya Sakai \\ Waseda University, Tokyo, Japan \\ tetsuyasakai@acm.org
}

\begin{abstract}
Some modern information access tasks such as natural language dialogue tasks are difficult to evaluate, for often there is no such thing as the ground truth: different users may have different opinions about the system's output. A few task designs for dialogue evaluation have been implemented and/or proposed recently, where both the ground truth data and the system's output are represented as a distribution of users' votes over bins on a non-nominal scale. The present study first points out that popular bin-by-bin measures such as Jensen-Shannon divergence and Sum of Squared Errors are clearly not adequate for such tasks, and that cross-bin measures should be used. Through experiments using artificial distributions as well as real ones from a dialogue evaluation task, we demonstrate that two cross-bin measures, namely, the Normalised Match Distance (NMD; a special case of the Earth Mover's Distance) and the Root Symmetric Normalised Order-aware Divergence (RSNOD), are indeed substantially different from the bin-by-bin measures. Furthermore, RSNOD lies between the popular bin-by-bin measures and NMD in terms of how it behaves. We recommend using both of these measures in the aforementioned type of evaluation tasks.
\end{abstract}

\section{KEYWORDS}

dialogue evaluation; earth mover's distance; evaluation measures; order-aware divergence; Wasserstein distance

\section{ACM Reference Format:}

Tetsuya Sakai. 2018. Comparing Two Binned Probability Distributions for Information Access Evaluation. In SIGIR '18: The 41st International ACM SIGIR Conference on Research and Development in Information Retrieval, fuly 8-12, 2018, Ann Arbor, MI, USA. ACM, New York, NY, USA, 4 pages. https://doi.org/10.1145/3209978.3210073

\section{INTRODUCTION}

Information access tasks have diversified; some of these tasks lack groud truth data. For example, if the system is required to estimate the user satisfaction score given a human-machine dialogue, it would be difficult to devise one ground truth score, for different people would rate the same dialogue differently. Moreover, if the system is aware of the above fact, it would be more useful if the system could output an estimated distribution of the users' ratings

Permission to make digital or hard copies of all or part of this work for personal or classroom use is granted without fee provided that copies are not made or distributed for profit or commercial advantage and that copies bear this notice and the full citation on the first page. Copyrights for components of this work owned by others than the author(s) must be honored. Abstracting with credit is permitted. To copy otherwise, or republish, to post on servers or to redistribute to lists, requires prior specific permission and/or a fee. Request permissions from permissions@acm.org.

SIGIR '18, fuly 8-12, 2018, Ann Arbor, MI, USA

(c) 2018 Copyright held by the owner/author(s). Publication rights licensed to ACM. ACM ISBN 978-1-4503-5657-2/18/07 . \$15.00

https://doi.org/10.1145/3209978.3210073 rather than a point estimate. In fact, even in traditional information retrieval, Maddalena et al. [6] have proposed to compute a distribution of $\mathrm{nDCG}$ scores instead of a point estimate, by preserving and utilising the different relevance assessments from different users.

The present study concerns tasks such as the Dialogue Breakdown Detection Challenge [2] and the NTCIR-14 STC-3 Dialogue Quality Subtask [8], where both the ground truth data and the system's output are represented as a distribution of users' opinions over bins on a non-nominal scale. We first point out that popular bin-by-bin measures such as Jensen-Shannon divergence and Sum of Squared Errors [5] are clearly not adequate for such tasks, and that cross-bin measures [7] should be used. While bin-by-bin measures simply accumulate the errors in each bin, cross-bin measures utilise the notion of the distance between bins. Through experiments using artificial distributions as well as real ones from a dialogue evaluation task, we demonstrate that two cross-bin measures, namely, the Normalised Match Distance (NMD) and the Root Symmetric Normalised Order-aware Divergence (RSNOD), are indeed substantially different from the bin-by-bin measures. Furthermore, RSNOD lies between the popular bin-by-bin measures and NMD in terms of how it behaves. As these two cross-bin measures behave somewhat differently, we recommend using both of them in evaluation tasks such as the ones mentioned above.

\section{PRIOR ART}

In the Dialogue Breakdown Detection Challenge (DBDC) [2], participating systems are given human-machine dialogues as input. Then, for each machine utterance, the systems are required to output an estimated probability distribution over three bins, namely, B (breakdown-which means that the system utterance is too inappropriate for the user to continue a meaningful conversation), PB (possible breakdown), and NB (not a breakdown). Note that the bins are ordinal, not nominal: for example, $\mathbf{B}$ is clearly "closer" to $\mathbf{P B}$ than to NB. Thirty assessors were hired to build the gold distribution over the three categories. DBDC currently uses, along with point estimate measures, Jensen-Shannon divergence and Mean Squared Error as the official evaluation measures. However, these bin-bybin measures cannot handle non-nominal bins properly. Perhaps to compensate for this, DBDC also computes the above measures after collapsing the three bins into two (e.g., by merging $\mathbf{B}$ and PB). However, a more elegant solution would be to utilise cross-bin measures [7], as we shall clarify in Section 3.

In the NTCIR-14 STC-3 Dialogue Quality subtask (STC-3 DQ), participating systems will be given a human-human, customerhelpdesk dialogue as input [8]; the systems are required to estimate the distribution of the assessors' ratings on that dialogue, in terms of customer satisfaction, task accomplishment, and so on [8], given a set of possible ratings (e.g., customer satisfaction on a 7-point 
scale). STC-3 DQ plans to build a gold distribution for each dialogue in a way similar to DBDC.

Sakai [8] observed that the probability bins for the STC-3 DQ are similar to those of DBDC in that they are non-nominal, and that neither Jensen-Shannon divergence nor Mean Squared Error is adequate for comparing the system and gold distributions. Hence, he proposed a cross-bin measure called the Symmetric Normalised Order-aware Divergence (SNOD) for the DQ subtask. However, he did not evaluate any of these measures. Moreover, he did not discuss a simple but important cross-bin measure called Match Distance [9], an instance of the Earth Mover's Distance, a.k.a. Wasserstein or Mallows Distance, which has proven to be highly useful in the context of computer vision and machine learning applications $[4,9]$.

\section{MEASURES}

We now formalise some measures for comparing two binned probability distributions, and explain why bin-by-bin measures are not adequate for our purpose. Let $A$ denote a given set of non-nominal bins, e.g., $A=\{\mathbf{B}, \mathbf{P B}, \mathrm{NB}\}$ for DBDC, and let $L=|A|$. Let $p(i)$ $(i=1, \ldots, L)$ denote the system's estimated probability for Bin $i$, so that $\sum_{i \in A} p(i)=1$. Similarly, let $p^{*}(i)$ denote the corresponding true probability, where $\sum_{i \in A} p^{*}(i)=1$. We also use the following vector notations: $p=(p(1), \ldots, p(L)), p^{*}=\left(p^{*}(1), \ldots, p^{*}(L)\right)$.

\subsection{Bin-by-Bin Measures}

Variational Distance (VD) [5], or Sum of Absolute Errors, forms the basis of the popular Mean Absolute Error:

$$
V D\left(p, p^{*}\right)=\sum_{i \in A}\left|p(i)-p^{*}(i)\right|
$$

However, measures based on VD are not good for our purpose. Consider a case with $L=5$, where $p^{*}=(0.2,0.2,0.2,0.2,0.2)$. It is clear that System $X$ that returns $p=(0.3,0.3,0.2,0.1,0.1)$ and $Y$ that returns $p=(0.3,0.3,0.2,0.1,0.1)$ will receive the same score $(V D=0.4)$, even though $X$, which returned a relatively flat distribution, should be considered better [8]. In this study, we shall use a normalised version of VD to ensure the $[0,1]$ range:

$$
\operatorname{NVD}\left(p, p^{*}\right)=\frac{V D\left(p, p^{*}\right)}{2} .
$$

Sum of Squares (SS), which forms the basis of Root Mean Squared Error, can handle this particular problem of VD:

$$
S S\left(p, p^{*}\right)=\sum_{i \in A}\left(p(i)-p^{*}(i)\right)^{2} .
$$

Let us consider a root normalised version:

$$
R N S S\left(p, p^{*}\right)=\sqrt{\frac{S S\left(p, p^{*}\right)}{2}} .
$$

For the above example, the RNSS for $X$ is 0.1414 , while that for $Y$ is 0.1732: $X$ is considered better. Chai and Draxler [1] discuss the advantages of Root Mean Squared Error (which is similar to RNSS) over Mean Absolute Error (which is similar to NVD).

Jensen-Shannon Divergence (JSD) is also free from the above problem of VD. Let $p_{M}(i)=\left(p(i)+p^{*}(i)\right) / 2$ for $i=1, \ldots, L$. JSD is defined as [5]:

$$
\mathcal{F S D}\left(p, p^{*}\right)=\frac{K L D\left(p \| p_{M}\right)+K L D\left(p^{*}|| p_{M}\right)}{2},
$$

(a)

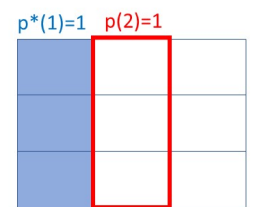

(c)

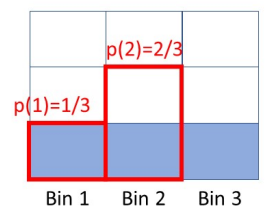

(b)

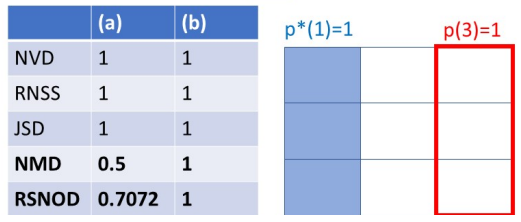

(d)

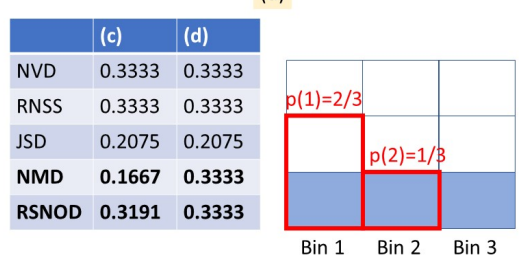

Figure 1: Examples to show the limitations of bin-by-bin measures (for $L=3$ ).

where the Kullback-Leibler divergence (KLD) for a pair of probability distributions $p_{1}, p_{2}$ is given by:

$$
K L D\left(p_{1} \| p_{2}\right)=\sum_{i \text { s.t. } p_{1}(i)>0} p_{1}(i) \log _{2} \frac{p_{1}(i)}{p_{2}(i)} .
$$

Note that JSD is symmetric (while KLD is not), but that it is not normalised. For the above example, the JSD for $X$ is 0.0390 while that for $Y$ is 0.0490 : again, $X$ is considered better.

Figure 1 provides some examples (with $L=3$ for simplicity) where all of the aforementioned bin-by-bin measures "fail." The bars shown in blue represent the gold probability distribution, and the red lines represent the estimated distribution; however, since the present study discusses symmetric measures only, the gold and system distributions may be swapped. It can be observed that, while Case (a) is better than Case (b) in that the estimated bin is closer to the true bin, none of the above measures can reflect this difference; similarly, while Case (c) is better than Case (d) in that it is less skewed towards the left, none of the above measures can reflect this difference either. It is clear that this problem arises from the simple summation across the bins: there is no notion of distance between bins.

\subsection{Cross-Bin Measures}

There is an existing measure that can look across bins, called Match Distance (MD) [9]. This is a special case of Earth Mover's Distance where the probabilities add up to one and the number of bins are a given [7]. Let $c p(i)=\sum_{k=1}^{i} p(k)$, and $c p^{*}(i)=\sum_{k=1}^{i} p^{*}(k)$. MD is just the sum of absolute errors computed from the cumulative probability distributions:

$$
M D\left(p, p^{*}\right)=\sum_{i \in A}\left|c p(i)-c p^{*}(i)\right|
$$

In the present study, we consider the following normalised version:

$$
N M D\left(p, p^{*}\right)=\frac{M D\left(p, p^{*}\right)}{L-1} .
$$

To see why $L-1$ is an appropriate normalisation factor, the reader should consider the worst case shown in Figure 1(b). Figure 1 shows that NMD correctly prefers Case (a) over (b), and prefers (c) over (d), despite its simplicity. 
Sakai [8] proposed a measure that considers the distance between a pair of bins more explicitly than NMD does. First, a distanceweighted sum of squares (DW) is defined for each bin:

$$
D W(i)=\sum_{j \in A}|i-j|\left(p(j)-p^{*}(j)\right)^{2} .
$$

Let $B^{*}=\left\{i \mid p^{*}(i)>0\right\}$, that is, the set of bins where the gold probabilities are positive. Order-Aware Divergence (OD) is the DW averaged over these non-empty gold bins:

$$
O D\left(p \| p^{*}\right)=\frac{1}{\left|B^{*}\right|} \sum_{i \in B^{*}} D W(i) .
$$

Similarly, let $B=\{i \mid p(i)>0\}$. Just as the symmetric JSD is obtained from KLD, Symmetric OD can be defined by swapping the system and gold distributions:

$$
S O D\left(p, p^{*}\right)=\frac{O D\left(p \| p^{*}\right)+O D\left(p^{*}|| p\right)}{2} .
$$

In this study, we define the Root Symmetric Normalised OD:

$$
R S N O D\left(p, p^{*}\right)=\sqrt{\frac{S O D\left(p, p^{*}\right)}{L-1}} .
$$

While Sakai did not take the square root, we prefer compensate for the fact that DW is a form of sum of squares: taking the square root boosts the absolute scores and stretches the lower end of the scale in the $[0,1]$ range. From Figure 1 , it is clear that RSNOD rates (a) higher than (b), and (c) higher than (d), just like NMD does.

\section{COMPARING THE MEASURES}

This section compares the aforementioned bin-by-bin measures, namely, NVD, RNSS, and JSD, and the cross-bin measures, namely, NMD and RSNOD. While the advantage of NMD and RSNOD over the bin-by-bin measures for the purpose of comparing two probability distributions over non-nominal bins is already clear, we seek to quantify the relationships among the five measures. Section 4.1 reports on an experiment with artificial distributions. Using artificial distributions does offer an advantage: if we just use real gold and system distributions from a real task, we cannot consider any other forms of distributions not covered by that particular task. In experiments with artifical distributions, we can consider a wide variety of distributions and their combinations. To complement the above, Section 4.2 reports on an experiment with real distributions from the latest DBDC task [2].

\subsection{Artificial Distributions}

To quantify the differences across the five measures, we can compute the measures for various pairs of probability distributions, rank the pairs by each measure, and compute Kendall's $\tau$. A high $\tau$ implies that the two measures behave similarly. To this end, we generated artificial probability distributions by random selection from a given set of bins, assuming that we have 30 votes (i.e., assessors) as in DBDC. We considered $L=3,5$, 7: for example, $L=3$ corresponds to the situation with DBDC where we have $\mathbf{B}, \mathbf{P B}$, and NB as the bins [2]; $L=7$ is applicable if the assessors are asked for (say) a dialogue satisfaction rating on a 7-point scale. We ensured that we have some distributions with zero-probability bins: for example, for $L=3$, we randomly created 20 distributions that cover

\begin{tabular}{|c|c|c|c|c|}
\hline \multicolumn{5}{|c|}{$L=3$ bins (60 distributions; 1,770 pairs of distributions) } \\
\hline & RNSS & JSD & NMD & RSNOD \\
\hline NVD & $\begin{array}{c}.947 \\
{[.942, .951]}\end{array}$ & $\begin{array}{c}.939 \\
{[.932, .947]}\end{array}$ & $\begin{array}{c}.773 \\
{[.759, .786]}\end{array}$ & $\begin{array}{c}.864 \\
{[.855, .874]}\end{array}$ \\
\hline RNSS & - & $\begin{array}{c}.899 \\
{[.892, .907]}\end{array}$ & $\begin{array}{c}.753 \\
{[.740, .767]}\end{array}$ & $\begin{array}{c}.859 \\
{[.850, .869]}\end{array}$ \\
\hline JSD & - & - & $\begin{array}{c}.734 \\
{[.719, .750]}\end{array}$ & $\begin{array}{c}.873 \\
{[.864, .882]}\end{array}$ \\
\hline NMD & - & - & - & $\begin{array}{c}.819 \\
{[.807, .831]}\end{array}$ \\
\hline \multicolumn{5}{|c|}{$L=7$ bins (140 distributions; 9,730 pairs of distributions) } \\
\hline & RNSS & JSD & NMD & RSNOD \\
\hline NVD & $\begin{array}{c}.845 \\
{[.842, .849]}\end{array}$ & $\begin{array}{c}.888 \\
{[.885, .890]}\end{array}$ & $\begin{array}{c}.593 \\
{[.585, .601]}\end{array}$ & $\begin{array}{c}.803 \\
{[.798, .808]}\end{array}$ \\
\hline RNSS & - & $\begin{array}{c}.808 \\
{[.804, .813]}\end{array}$ & $\begin{array}{c}.602 \\
{[.594, .610]}\end{array}$ & $\begin{array}{c}.815 \\
{[.810, .820]}\end{array}$ \\
\hline JSD & - & - & $\begin{array}{c}.568 \\
{[.559, .576]}\end{array}$ & $\begin{array}{c}.801 \\
{[.797, .806]}\end{array}$ \\
\hline NMD & - & - & - & $\begin{array}{c}.643 \\
{[.635, .650]}\end{array}$ \\
\hline
\end{tabular}
all three bins, and another 20 that cover only two bins, and another
Table 1: Ranking pairs of distributions: Kendall's $\tau$ with 95\%CIs.

20 that cover only one bin. Hence, for $L=3$, we created $20 * 3=60$ different distributions in total, which gives us $60 * 59=1,770$ pairs of distributions to compute the measures and thereby rank the pairs. Similarly, for $L=5$, we created $20 * 5=100$ distributions $(4,950$ pairs); for $L=7$, we created $20 * 7=140$ distributions (9,730 pairs). Due to lack of space, however, we will not discuss $L=5$ any further.

Table 1 shows the kendall's $\tau$ values with $95 \%$ confidence intervals: they represent the similarity between each pair of measures in terms of how they rank the different pairs of artificial distributions. The absolute $\tau$ values are not so meaningful here, since our randomly generated distributions are probably not representative of real gold and system distributions; what matters is the relative behaviour of $\tau$ across different pairs of measures. A trend that is consistent across the different $L$ 's is that the $\tau$ 's concerning the three bin-by-bin measures and RSNOD (e.g., .859-.947 for $L=3$ ) are higher than the $\tau$ between NMD and RSNOD (e.g., .819 for $L=3$ ), which in turn is higher than the $\tau$ 's between NMD and binby-bin measures (e.g., .734-.773 for $L=3$ ). That is, NMD behaves substantially differently from the bin-by-bin measures, and RSNOD lies somewhere in between NMD and the bin-by-bin measures (and closer to the latter) in terms of how it ranks different pairs of binned distributions.

\subsection{Real Distributions}

We also utilised real data from the latest DBDC task [2]: the task provides a gold distribution over $L=3$ bins (NB, PB, B) for a total of 2,000 system utterances. In addition, we obtained two runs from a top performing team in the English subtask [3]; hereafter, the team's least and most effective runs according to the official results are referred to as System 1 and System 2, respectively. Thus, we have 2,000 estimated distributions from each of these systems.

Table 2(I) shows the Kendall's $\tau$ results in a way similar to Table 1: these new results are based on ranking the 2,000 utterances by each measure, computed by comparing System 2's distribution with the 
Table 2: Comparing the measures with the 2,000 utterances from the DBDC3 data.

\begin{tabular}{|c|c|c|c|c|}
\hline \multicolumn{5}{|c|}{ (I) Kendall's $\tau$ with 95\%CIs (System 2) } \\
\hline & RNSS & JSD & NMD & RSNOD \\
\hline NVD & $\begin{array}{c}.960 \\
{[.959, .962]}\end{array}$ & $\begin{array}{c}.900 \\
{[.892, .908]}\end{array}$ & $\begin{array}{c}.799 \\
{[.790, .807]}\end{array}$ & $\begin{array}{c}.946 \\
{[.943, .949]}\end{array}$ \\
\hline RNSS & $\begin{array}{l}- \\
-\end{array}$ & $\begin{array}{c}.912 \\
{[.904, .920]}\end{array}$ & $\begin{array}{c}.795 \\
{[.786, .804]}\end{array}$ & $\begin{array}{c}.960 \\
{[.958, .962]}\end{array}$ \\
\hline JSD & - & - & $\begin{array}{c}.792 \\
{[.781, .802]}\end{array}$ & $\begin{array}{c}.915 \\
{[.907, .922]}\end{array}$ \\
\hline NMD & - & - & - & $\begin{array}{c}.832 \\
{[.825, .840]}\end{array}$ \\
\hline \multicolumn{5}{|c|}{ (II) Preference agreement for comparing Systems 1 and 2} \\
\hline & RNSS & JSD & NMD & RSNOD \\
\hline NVD & $96.1 \%$ & $95.3 \%$ & $89.4 \%$ & $95.5 \%$ \\
\hline RNSS & - & $97.8 \%$ & $89.4 \%$ & $98.2 \%$ \\
\hline JSD & - & - & $89.9 \%$ & $98.2 \%$ \\
\hline NMD & - & - & - & $90.8 \%$ \\
\hline
\end{tabular}

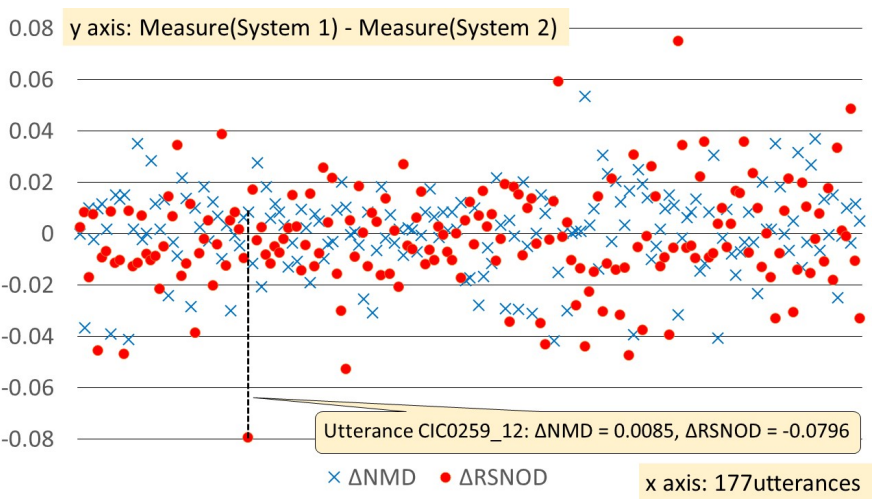

Figure 2: 177 true disagreements (out of 2,000 utterances) between NMD and RSNOD regarding Systems 1 and 2.

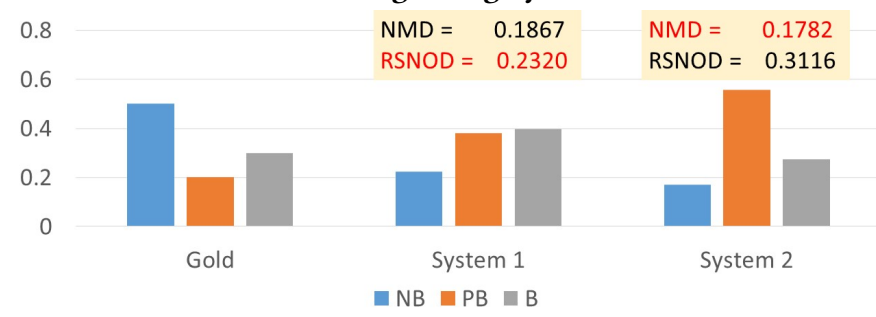

Figure 3: DBDC probability distributions of Gold, System 1, and System 2 for Utterance CIC0259_12.

gold one. (Similar results with System 1 are omitted due to lack of space.) It is clear that even with real distributions from a dialogue task, the aforementioned observation (Section 4.1) still holds.

Table 2(II) compares the five measures in a different way. For each of the 2,000 utterances, the score delta between Systems 1 and 2 is computed for each measure; then we count the preference agreement between two measures. For example, if both NMD and RSNOD agree that System 1's distribution for a particular utterance is better, that is an agreement. Between these two cross-bin measures, there were 1,816 agreements $(1816 / 2000=90.8 \%$ as shown in Table 2(II)). It can be observed that the trend is similar to the results with $\tau$ 's: the bin-by-bin measures and RSNOD are similar to each other (95.398.2\% agreement); NMD and RSNOD are less similar (90.8\%); and NMD is even less similar to the bin-by-bin measures (89.4-89.9\%).

Of the $2000-1816=184$ disagreeements between NMD and RSNOD, the delta for NMD was zero (i.e., Systems 1 and 2 were considered equivalent) for eight utterances, and hence 177 were "true" disagreements. Figure 2 visualises these instances: it can be observed that although NMD and RSNOD disagreed in terms of the sign of the delta, the magnitute of the delta is less than 0.08 in every case. Thus, these are not serious disagreements. Figure 3 zooms in on an utterance indicated in Figure 2: for this utterance, RSNOD says that System 1 is better, while NMD says that System 2 is better. Even for us humans, it may be difficult to say which is correct; it may not matter. Hence, our recommendation for evaluation tasks based on comparing binned distributions would be to use both NMD and RSNOD, but not the bin-by-bin measures.

Finally, we conducted a randomisation test for the difference between Systems 1 and 2 for every measure. All five measures agree that System 2 is better, and the $p$-values for NVD, RNSS, JSD, NMD, RSNOD are 0.182, 0.140, 0.139, 0.149, 0.143. Hence NMD and RSNOD appear to be similar in terms of discriminative power as well, although a more extensive investigation is required.

\section{CONCLUSIONS AND FUTURE WORK}

We clarified the advantages of cross-bin measures over the popular bin-by-bin measures for the purpose of comparing binned distributions in an evaluation task. Our future work includes further investigation into the differences between NMD and RSNOD in terms of discriminative power and agreement with human perception. The data used in our experiments are publicly available for replication by other researchers ${ }^{1}$.

\section{REFERENCES}

[1] T. Chai and R.R. Draxler. 2014. Root Mean Square Error (RMSE) or Mean Absolute Error (MAE)? - Arguments against avoiding RMSE in the Literature. Geoscientific Model Development 7 (2014), 1247-1250.

[2] Ryuichiro Higashinaka, Kotaro Funakoshi, Michimasa Inaba, Yuiko Tsunomori, Tetsuro Takahashi, and Nobuhiro Kaji. 2017. Overview of Dialogue Breakdown Detection Challenge 3. In Proceedings of Dialog System Technology Challenge 6 (DSTC6) Workshop.

[3] Sosuke Kato and Tetsuya Sakai. 2017. RSL17BD at DBDC3: Computing Utterance Similarities based on Term Frequency and Word Embedding Vectors. In Proceedings of DSTC6. http://workshop.colips.org/dstc6/papers/track3_paper13_kato.pdf

[4] Elizaveta Levina and Peter Bickel. 2001. The Earth Mover's Distance is the Mallows Distance: Some Insights from Statistics. In Proceedings of ICCV 2001. 251-256.

[5] Jianhua Lin. 1991. Divergence Measures Based on the Shannon Entropy. IEEE Transactions on Information Theory 37, 1 (1991), 145-151.

[6] Eddy Maddalena, Kevin Roitero, Gianluca Demartini, and Stefano Mizzaro. 2017. Considering Assessor Agreement in IR Evaluation. In Proceedings of ACM ICTIR 2017. 75-82.

[7] Yossi Rubner, Carlo Tomasi, and Leonidas J. Guibas. 2000. The Earth Mover's Distance as a Metric for Image Retrieval. International fournal of Computer Vision 40, 2 (2000), 99-121.

[8] Tetsuya Sakai. 2017. Towards Automatic Evaluation of Multi-Turn Dialogues: A Task Design that Leverages Inherently Subjective Annotations. In Proceedings of EVIA 2017. 24-30.

[9] Michael Werman, Shmuel Peleg, and Azriel Rosenfeld. 1985. A Distance Metric for Multidimensional Histograms. Computer Vision, Graphics, and Image Processing 32 (1985), 328-336.

\footnotetext{
${ }^{1}$ http://waseda.box.com/sigir2018short
} 